www.nature.com/leu

\title{
REVIEW
}

\section{Bisphosphonates as antimyeloma drugs}

\author{
ND Modi and S Lentzsch \\ Department of Medicine, Division of Hematology/Oncology, University of Pittsburgh, Pittsburgh, PA, USA
}

In patients with symptomatic multiple myeloma (MM), bisphosphonate (BP) treatment has been widely used to prevent bone loss and preserve skeletal health because of its proven effects on inhibiting osteoclast-mediated bone resorption. In addition to their effects on osteoclasts, it is becoming increasingly evident that BPs may have additional effects on the bone microenvironment and cells other than osteoclasts that may potentially inhibit the development and progression of $M M$. This review focuses on the pathophysiology of MM with an emphasis on the events that drive MM progression within the bone and the mechanisms by which BPs may inhibit specific processes. The underlying molecular mechanisms that drive the modulation of cellular fate and function and consequent physiological outcomes are described. Direct effects on myeloma cell growth and survival and the interactions between myeloma cells and the bone microenvironment are discussed. Clinical evidence of the antimyeloma effects of BPs is emerging and is also reviewed.

Leukemia (2012) 26, 589-594; doi:10.1038/leu.2011.282;

published online 18 October 2011

Keywords: bisphosphonates; multiple myeloma; pamidronate; survival; zoledronic acid

\section{Introduction}

Monoclonal gammopathy of unknown significance is an asymptomatic condition characterized by the clonal expansion of plasma cells. In a small proportion of patients, monoclonal gammopathy of unknown significance progresses through a multistage process to symptomatic and malignant multiple myeloma (MM). There are few somatic genetic differences between the asymptomatic monoclonal gammopathy of unknown significance and malignant MM. Interactions between myeloma cells and bone cells and the extracellular matrix proteins within the bone microenvironment underlie this progression and are mediated through cell surface receptors - for example, integrins, cadherins, selectins, syndecans and the immunoglobulin superfamily of cell adhesion molecules. ${ }^{2}$ These interactions trigger a self-amplifying cascade of events that result in the secretion of cytokines and growth factors (such as vascular endothelial growth factor (VEGF), insulin-like growth factor-1, interleukin-6 (IL-6) and IL-1 $\beta$ ) and members of the tumor necrosis factor superfamily (TGF $\beta 1$, CCL3, hepatocyte growth factor and IL-10) that promote the growth and proliferation of myeloma cells, increase bone resorption and enhance drug resistance by inducing antiapoptotic pathways. ${ }^{1,2}$ The underlying mechanisms and pathways driving this vicious cycle of tumor growth and bone destruction have been extensively reviewed. ${ }^{1,3-5}$

Correspondence: Dr S Lentzsch, Division of Hematology/Oncology, University of Pittsburgh, UPMC Cancer Pavilion, 5150 Centre Avenue, Suite 568, Pittsburgh, PA 15232, USA.

E-mail: lentzschs@upmc.edu

Received 11 May 2011; revised 18 August 2011; accepted 6 September 2011; published online 18 October 2011
Bisphosphonates (BPs) are degradation-resistant analogs of inorganic pyrophosphate that can inhibit bone resorption. This effect is of particular clinical relevance in patients with symptomatic MM who, as a consequence of the physiological changes induced by malignant bone disease, undergo profound bone loss. Newer generation, nitrogen-containing BPs (N-BPs) prevent bone loss because of cellular effects involving both apoptosis of the osteoclasts and the destruction of the osteoclastic cytoskeleton, resulting in decreased osteoclast activity. The biochemical basis of these effects for N-BPs (for example, alendronate, risedronate, ibandronate, pamidronate and zoledronic acid) is the inhibition of farnesyl pyrophosphate synthase (FPPS), a key branch-point enzyme in the mevalonate pathway. 6,7 Consequently, cellular availability of isoprenoid lipids such as farnesyl pyrophosphate and geranylgeranyl pyrophosphate is decreased and the post-translational modification and function of small GTP-binding proteins that modulate key signaling events involved in cell survival, differentiation and proliferation are impaired. ${ }^{8-10}$ Experiments in several in-vitro and in-vivo experimental model systems of cancer in general and MM in particular suggest that BPs may negatively modulate promyeloma signaling events and thereby provide clinical benefits that extend beyond bone conservation. This review examines the mechanisms by which BPs may interfere with progression of MM

\section{Preclinical evidence and molecular basis of antimyeloma effects of BPs}

Several preclinical studies have provided strong evidence for the antimyeloma potential of BPs (Figure 1). ${ }^{2,11-18}$ In a study by Baulch-Brown et $\mathrm{al}_{,}{ }^{19}$ the authors showed that myeloma cells treated with zoledronic acid, or other mevalonate pathway antagonists such as fluvastatin (an HMG-CoA reductase inhibitor) or SCH66336 (a farnesyl transferase inhibitor) suppressed the proliferation of myeloma cells (RPMI 8226, U266, OMP2, LP1 and $\mathrm{NCl}-\mathrm{H} 929)$ and that combinations of zoledronic acid and fluvastatin, but not zoledronic acid and SCH66336 acted synergistically. The study also showed that the antiproliferative effect of mevalonate pathway inhibitors is mediated principally by prevention of geranylgeranylation and is the result of both cell-cycle arrest and apoptosis induction. Indeed, microarray and quantitative real-time PCR analyses further demonstrated that genes related to apoptosis, cell-cycle control and the mevalonate pathway were particularly affected by zoledronic acid and fluvastatin, and that some of these transcriptional effects were synergistic. Similarly, incadronate and mevastatin (a known inhibitor of the mevalonate pathway) caused apoptosis in JJN-3 myeloma cells and inhibited cell proliferation. ${ }^{20}$ Moreover, mevalonate pathway intermediates such as geranylgeraniol and farnesol prevented incadronateinduced apoptosis of JJN-3 myeloma cells and had a partial 


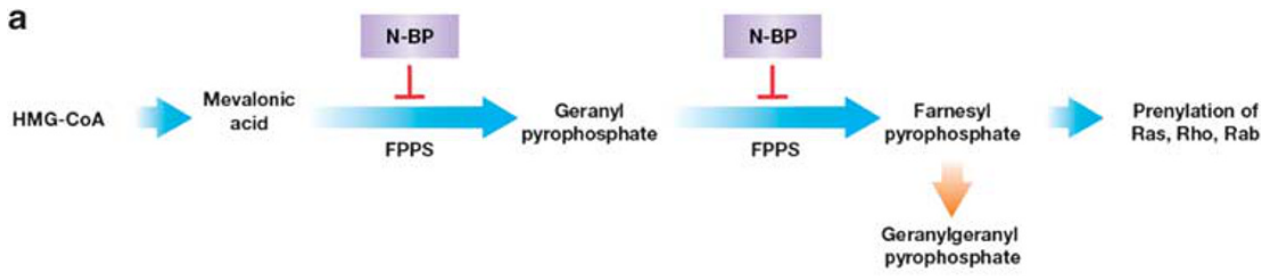

b

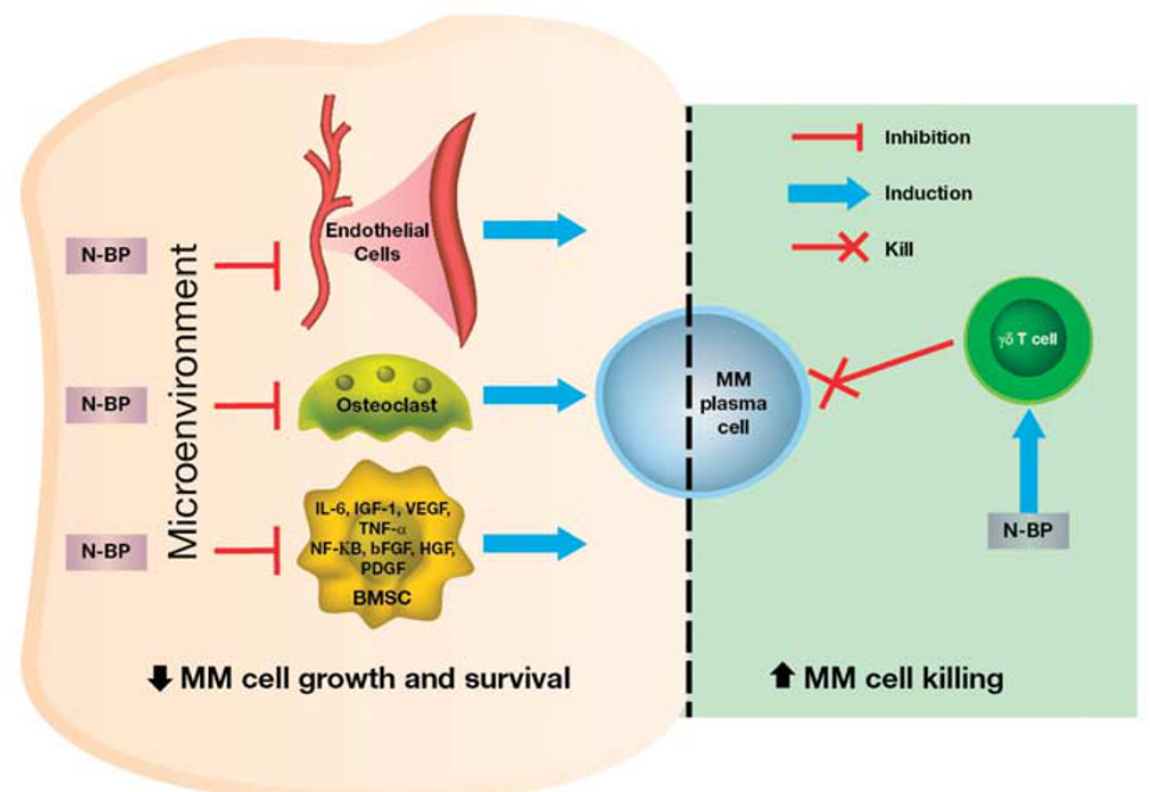

Figure $1 \mathrm{~N}$-BPs may modulate myeloma progression through their effects on the mevalonate pathway and directly on MM plasma cells, bone marrow cells and immune cells in the bone marrow. (a) N-BPs affect cell survival by blocking the key enzyme (that is, FPPS) in the mevalonate pathway required for prenylation of proteins. ${ }^{11,12,14}(\mathbf{b})$ N-BPs prevent proliferation of MM plasma cells directly via inhibition of growth factors that promote cell growth and survival within the bone microenvironment and indirectly via inhibition of angiogenesis (left). ${ }^{2,14-18} \mathrm{~N}$-BPs enhance host antitumor immune response (right). ${ }^{13}$ The dotted line depicts the boundary between the bone microenvironment and the extraskeletal vasculature. bFGF, fibroblast growth factor; FPPS, farnesyl pyrophosphate synthase; HGF, hepatocyte growth factor; IGF-1, insulin-like growth

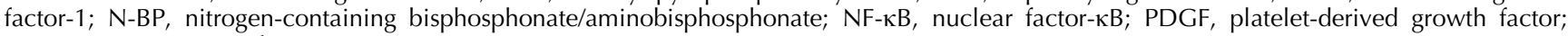
TNF- $\alpha$, tumor necrosis factor- $\alpha$.

effect on cell-cycle arrest, suggesting that the anticancer effects of incadronate are a direct consequence of its known mechanism of action (that is, mevalonate pathway inhibition).

In a study by Shipman et $a^{21}$ in the human myeloma cell line JJN-3 and the EBV transformed Burkitt lymphoma cell line HS-Sultan, ${ }^{22}$ pamidronate and the more potent BP, YM175, induced apoptosis and significantly decreased cell number $(P<0.001)$. Both pamidronate and YM175 increased in the proportion of cells with altered nuclear morphology $(P<0.05)$ and fragmented DNA, in both JJN-3 and HS-Sultan cells. In contrast, clodronate, a BP that does not contain any nitrogen and does not inhibit the mevalonate pathway, had little effect on cell number and did not cause apoptosis at the concentrations examined. In another study, risedronate dose dependently inhibited prenylation of (ras in brain) RAB GTPases (for example, Rap1A and Rab6), eliciting a dose-dependent apoptotic response in human myeloma cells and arresting these cells in the $\mathrm{S}$ phase. $^{23}$ This study also showed that geranylgeraniol prevented inhibition of prenylation, induction of apoptosis and cell-cycle arrest in response to risedronate. These data support the critical role of mevalonate pathway inhibition by $\mathrm{N}-\mathrm{BPs}$ and the consequent impairment of function of small GTPases in mediating the observed anticancer effects (Figure 1a).

The role of isoprenylation of proteins in mediating these events is also supported by data from a more recent study in the 5T2MM model of MM that showed that 3-PEHPC (2-[3- pyridinyl]-1-hydroxyethylidene-1,1-phosphonocarboxylic acid), a novel geranylgeranyl-transferase II inhibitor, prevented bone loss, inhibited the development of osteolytic bone lesions and reduced myeloma burden in bone. ${ }^{24}$ In contrast to other agents that inhibit the mevalonate pathway or post-translational prenylation, BPs have a natural affinity toward bone mineral that limits the effects of mevalonate pathway inhibition to select cellular components (mainly osteoclasts, and non-osteoclast cells including tumor cells and lymphocytes) within the bone microenvironment. This is particularly desirable in a disease such as MM, a disease whose progression is primarily driven within the bone.

In addition to data from cell-culture experiments, in-vivo experiments in animal models of $M M$ provide additional evidence of the antimyeloma activity of BPs. For example, zoledronic acid significantly prolonged survival in severe combined immunodeficiency mice inoculated with human INA-6 plasma cells. ${ }^{12}$ Importantly, this study used clinically relevant doses of zoledronic acid, and histological analysis of INA-6 tumors from the peritoneal cavity revealed extensive areas of apoptosis associated with poly (ADP ribose) polymerase cleavage. Furthermore, western blot analysis of tumor homogenates demonstrated the accumulation of unprenylated Rap1A, which is indicative of the uptake of zoledronic acid by non-skeletal tumors and inhibition of the mevalonate pathway. 
Similarly, in another in-vivo study, zoledronic acid prevented the formation of skeletal lesions, prevented cancellous bone loss and loss of bone mineral density, and reduced osteoclast perimeter in mice injected with 5T2MM murine myeloma cells. ${ }^{25}$ Zoledronic acid also decreased paraprotein concentration, decreased tumor burden and reduced angiogenesis. In separate experiments, Kaplan-Meier analysis demonstrated a significant increase in disease-free survival after treatment with zoledronic acid when compared with control $(P<0.005)$. Thus, these data are consistent with the antimyeloma effects observed with other mevalonate pathway inhibitors.

As a consequence of the inhibition of the mevalonate pathway in cells, N-BPs can induce formation of a novel ATP analog, triphosphoric acid 1-adenosin-5'-yl ester 3-(3-methylbut-3-enyl) ester or Apppl. ${ }^{26}$ Accumulation of Apppl correlated with the capacity of N-BPs to inhibit the mevalonate pathway in macrophages. Apppl inhibited the mitochondrial ADP/ATP translocase in isolated rat liver mitochondria and caused apoptosis in osteoclasts. These data broaden the mechanistic basis of N-BPs' action (beyond inhibition of post-translational modification of small GTPases involved in cell signaling) to include Apppl formation. In contrast to mevalonate pathway inhibition that results in impaired function of small GTPases, Apppl functions through the blockade of mitochondrial ADP/ATP translocase, thereby inducing apoptosis. In addition to its effects on mitochondrial function, Apppl and another intermediate, isopentenyl pyrophosphate (IPP), are phosphoantigens that can enhance host anticancer activity.

Raikkonen et $\mathrm{a}^{27}$ reported that zoledronic acid-induced IPP/Apppl accumulation in MCF-7 breast cancer cells was decreased by farnesol and almost completely blocked by geranylgeraniol and geranyl pyrophosphate. The functionality of the regulatory enzymes of IPP and Apppl, IPP isomerase and aminoacyl-tRNA synthase, respectively, or protein levels of FPPS were not affected by the treatments. However, protein levels of 3-hydroxy-3-methylglutaryl-coenzyme A reductase (the rate-controlling enzyme of the mevalonate pathway) and unprenylated Rap1A were decreased, thereby suggesting that mevalonate pathway intermediates may also rescue N-BP effects by inhibiting IPP/Apppl accumulation. ${ }^{27}$ Thus, the anticancer effects of mevalonate pathway inhibition result from multiple outcomes including impairment of function of small GTPases, inhibition of ATP/ADP translocase and stimulation of host antitumor activity.

\section{Synergistic activity of BPS with other agents and antimyeloma therapies}

Sequential blockage of the mevalonate pathway by zoledronic acid and simvastatin resulted in the synergistic induction of apoptosis and reversal of cell adhesion-mediated drug resistance. ${ }^{28}$ Treatment of myeloma cell lines with the combination of zoledronic acid and dexamethasone also demonstrated synergistic induction of apoptosis in vitro, providing a rationale for potential applications in vivo. ${ }^{29,30}$ In another study, bortezomib and zoledronic acid showed distinct and synergistic inhibitory effects on cell proliferation, adhesion, migration and expression of angiogenic cytokines (for example, VEGF, bFGF, hepatocyte growth factor and platelet-derived growth factor). ${ }^{14}$ Similar effects were observed on capillarogenic organization and expression of vascular markers in cells that became vasculogenic. Activation of VEGFR2, ERK1/2 and NF- $\kappa B$ activity was also inhibited. Overall, these data provide evidence that the exposure of bone marrow macrophages in MM during treatment with zoledronic acid impacts their angiogenic and vasculogenic properties (Figure 1b).

\section{BPs as immunomodulators}

In addition to their direct effects on cancer and bone cells, BPs may also repress cancer progression by stimulating the host anticancer response and/or by inhibiting proangiogenic signaling by immune cells. Newer generation BPs appear to induce or activate $\mathrm{V} \gamma 9 \mathrm{~V} \delta 2$ subset $\mathrm{T}$ cells by mimicking phosphoantigens and/or by increasing circulating phosphoantigen levels. ${ }^{31}$ The $\mathrm{V} \gamma 9 \mathrm{~V} \delta 2 \mathrm{~T}$ cells have antitumor activity and appropriate cell surface antigens to target secondary lymphoid organs and exert costimulatory activity. ${ }^{32}$ BP treatment has been shown to induce the accumulation of IPP and dimethylallyl bisphosphonate, phosphoantigens that activate $\mathrm{V} \gamma 9 \mathrm{~V} \delta 2 \mathrm{~T}$ cells. $^{13}$ Several studies have demonstrated that zoledronic acid treatment causes the expansion of $\mathrm{V} \gamma 9 \mathrm{~V} \delta 2 \mathrm{~T}$-cell populations and increases sensitivity of cancer cells to the cytotoxic effects of V $\gamma 9 \mathrm{~V} \delta 2 \mathrm{~T}$ cells. ${ }^{33,34}$ In a small clinical study, patients infused with zoledronic acid-activated $\mathrm{V} \gamma 9 \mathrm{~V} \delta 2 \mathrm{~T}$ cells generated from the culture of peripheral blood mononuclear cells maintained their M-protein levels in the serum at baseline, demonstrating that activation of the innate host antitumor response by N-BPs can successfully control the expansion of myeloma cells. ${ }^{35}$

In MM, cells belonging to the monocyte-macrophage lineage form part of an inflammatory circuit that promotes tumor progression, invasion and metastasis mainly through their proangiogenic activity. ${ }^{16,18}$ These cells express a broad array of matrix metalloproteases, proangiogenic cytokines and growth factors. The combination of factors facilitates matrix breakdown and tunneling that promote neovascularization and invasion. A recent study evaluating the effect of clinically relevant doses of zoledronic acid on bone metastasis in a constitutionally active ErbB-2 transgenic mouse model reported that zoledronic acid treatment resulted in M2 (anti-inflammatory and proangiogenic) to M1 (antitumor) reversion of tumor-associated macrophages. ${ }^{36}$ This study also reported that zoledronic acid treatment resulted in a profound reduction in $\mathrm{CD} 11^{+}$macrophages infiltrating mammary lesions and was accompanied by reduced vascularization of the tumor and decreased VEGF levels in the tumor microenvironment. Moreover, this study also showed that Ras prenylation and Ras-GTP levels were suppressed by treatment with zoledronic acid. Overall, the study demonstrated that zoledronic acid improved disease-free and overall survival (OS). These data are consistent with decreases in VEGF levels observed in cancer patients treated with repeated low-dose therapy with zoledronic acid. ${ }^{17}$ These results show that $\mathrm{N}$-BPs affect the immune system by both increasing host antitumor activity and reducing tumor-associated neovascularization.

\section{$B P$ effects on the bone marrow microenvironment}

In $\mathrm{MM}$, bone marrow stromal cells increase the concentration of angiogenic factors and matrix degrading enzymes in the bone marrow microenvironment by direct secretion or by the stimulation of myeloma cells or endocrine cells through paracrine interactions. ${ }^{15,16}$ In-vitro studies have demonstrated the anticancer potential of zoledronic acid on myeloma cell lines, but few data are available on its effects on bone marrow stromal cells. ${ }^{37}$ In a study by Corso et al, ${ }^{37}$ treatment of bone marrow stromal cells derived from the bone marrow of myeloma patients with zoledronic acid reduced proliferation; increased apoptosis; decreased IL-6, tumor necrosis factor- $\alpha$ and IL-1 $\beta$ production; and modified the pattern of expression of adhesion molecules, especially those involved in plasma cell binding. These effects on bone marrow stromal cells suggest that the anticancer activity of zoledronic acid may in part result from its 
ability to disrupt the vicious cycle of signaling that promotes myeloma growth and progression (Figure $1 \mathrm{~b}$ ).

Taken together, the data available suggest that BPs inhibit MM growth directly (Figure 1a) and indirectly via the bone marrow microenvironment or by stimulating the immune system (Figure $1 \mathrm{~b}$ ). The final mechanism responsible for a possible antimyeloma effect is not fully understood, but it seems that indirect effects via modulation of the microenvironment mediate a broader antimyeloma effect.

\section{Clinical evidence of the antimyeloma effect of BPs}

Data from clinical trials of BPs in patients with $\mathrm{MM}^{38-40}$ provided the first clinical evidence of antimyeloma activity of BPs. For example, in a long-term follow-up (8.6 years) of a placebo-controlled trial $(N=619)$, the subset of clodronatetreated patients who did not have vertebral fractures at baseline had significantly longer OS vs patients who received placebo (median OS, 59 months vs 37 months, respectively; $P=0.006) .{ }^{40}$ Similarly, in patients with newly diagnosed or relapsed/refractory MM $(N=392)$, long-term treatment with pamidronate significantly increased survival in the subset of patients with $M M$ receiving second-line antimyeloma therapy 14 months vs 21 months; $P=0.041$ compared with placebo. ${ }^{39}$ In a retrospective analysis of patients with $M M$ who had bone marker assessments $(N=353)$ in a phase III trial comparing zoledronic acid (4 mg) with pamidronate $(90 \mathrm{mg})$, patients with high baseline bone-specific alkaline phosphatase ( $\geqslant 146 \mathrm{IU} / \mathrm{I})$ levels had significantly better 25-month survival with zoledronic acid than with pamidronate (82 vs 53\%, respectively; $P=0.041)^{38}$

Small clinical studies have also provided insight into the antimyeloma potential of zoledronic acid. In 2007, Aviles et al conducted a clinical trial in which 94 patients (treated with cyclophosphamide, vincristine, melphalan and prednisone) were randomized to receive either zoledronic acid $(4 \mathrm{mg}$ intravenous infusion every 28 days) or not (control group). After 49.6 months median follow-up, assessment of the primary end points of 5-year event-free survival and 5-year OS showed significantly greater benefit for the zoledronic acid-treated group vs the control group (5-year event-free survival was $80 \%$ in the zoledronic acid group vs $52 \%$ in the control group $(P<0.01)$; 5 -year OS was $80 \%$ in the zoledronic acid group vs $46 \%$ in the control group $(P<0.01)){ }^{41}$

Recently, the Medical Research Council Myeloma IX trial, a large, randomized, controlled trial to evaluate the role of BPs in patients with newly diagnosed MM, was designed and conducted by the UK Medical Research Council. ${ }^{42}$ This phase III trial $(N=1960)$ compared the efficacy and safety of monthly intravenous zoledronic acid $(4 \mathrm{mg})$ vs daily oral clodronate (1600 mg) used concurrently with the prevailing standard treatment. The primary end point of this trial was OS, and additional end points included progression-free survival, response rates and incidence of skeletal-related events. Patients were assigned to two main treatment pathways based on their age and performance status. Younger, transplant-eligible patients were assigned to the intensive pathway, which consisted of induction therapy (randomized between standard cytotoxic and thalidomide-based regimens) followed by autologous stem-cell transplantation. Non-transplant eligible patients were assigned to the non-intensive pathway, which consisted of systemic therapy only (randomized between melphalan/prednisone and a thalidomide-based regimen). Within each treatment pathway, patients were randomized to oral clodronate (1600 mg/day) or zoledronic acid (4 mg via $15 \mathrm{~min}$ intravenous infusion) and treated until disease progression or death.

Of 1960 evaluable patients, 981 received zoledronic acid and 979 received clodronate. At the median follow-up of 3.7 years, $24 \%$ and $19 \%$ of patients, respectively, discontinued study before disease progression. Median time on treatment was $\sim 1$ year across all treatment groups. At the time of the database lock, $11-13 \%$ of patients (zoledronic acid and clodronate arms, respectively) were still receiving BP treatment. Zoledronic acid significantly prolonged both progression-free survival and OS $(P=0.0179$ and $P=0.0118$, respectively) vs clodronate. But it should be pointed out that the effect of zoledronic acid relative to clodronate on OS and progression-free survival occurred in the first 4 months, whereas OS and progression-free survival curves were essentially parallel during follow-up. This is probably due to the markedly higher relative activity of zoledronic acid vs clodronate also shown in a rat osteoclast model. ${ }^{43}$ In addition, the better bioavailability of zoledronic acid due to intravenous application possibly results in a stronger inhibition of FPPS and subsequent protein prenylation. ${ }^{44}$ Zoledronic acid also reduced the proportion of patients with a skeletal-related event vs clodronate $(27.0$ vs $35.3 \%$, respectively; $P=0.0004)$. Further, the improvement in OS was maintained after adjustment for time to first skeletal-related event in a Cox model $(P=0.0178)$, suggesting that antimyeloma effects likely underlie the OS benefit. In addition, zoledronic acid halved the incidence of new osteolytic lesions, regardless of treatment pathway. ${ }^{42}$ These data support an antimyeloma benefit from zoledronic acid treatment in patients with newly diagnosed MM.

In contrast to these studies demonstrating the antimyeloma benefits of BPs, there are also reports that suggest that BPs do not have any clinically meaningful effect on the progression of MM. For example, in an updated indirect meta-analysis of 17 trials with $\sim 3000$ patients, ${ }^{45}$ the authors concluded that BP treatment was not significantly associated with any survival benefit. However, it should be noted that this study included several different BPs, which in individual studies have shown differences in their antimyeloma activity. Overall, zoledronic acid and pamidronate have proven to be more effective than other BPs in terms of the clinical benefit they provide. Moreover, it should be noted that these analyses did not include the Medical Research Council Myeloma IX study ${ }^{42}$ and that although it did include the study by Aviles et $a l^{41}$ that showed survival benefit, it comprised only a small fraction of the pooled population and the effect might therefore be masked. Similarly, in a study by McCloskey et al, ${ }^{40,46}$ survival benefit was not observed in the overall study population, but subgroup analysis showed significant survival benefit in the subset of patients without skeletal involvement at diagnosis. Finally, in the study by Musto et al, ${ }^{47}$ zoledronic acid treatment did not have an effect on the progression of asymptomatic MM to symptomatic disease. Taken together, these data suggest that to gain clinically meaningful insight into the question of antimyeloma benefit, one may need to evaluate data not only in the context of a specific type of BP but also in the disease stage.

\section{Discussion}

BPs have an important role in the treatment of MM bone disease. In addition to the established benefit to skeletal health, there are both in-vitro and in-vivo evidence that BPs have 
potential antimyeloma effects. For example, Tassone et a ${ }^{29}$ showed that combination of zoledronic acid and dexamethasone inhibits growth and induces apoptotic death synergistically in $M M$ cell lines. Ural et $a l^{30}$ extended the observation of zoledronic acid synergy with antimyeloma agents in their study demonstrating cytotoxic effects of zoledronic acid in combination with dexamethasone and thalidomide on myeloma cell lines ARH-77 (EBV transformed) and RPMI-8226. Croucher et $\mathrm{a}^{25}$ used zoledronic acid on 5T2MM-bearing mice, and results showed that N-BPs decreased osteolysis, tumor burden, and angiogenesis and increased survival.

Bone resorption in $\mathrm{MM}$ releases several types of growth factors and cytokines including VEGF, tumor necrosis factor and interleukins; all of which further support the tumor growth. In addition to the direct effects of BPs on myeloma cells, BPs may indirectly affect tumor growth by preventing bone resorption and inhibiting further release of tumor stimulating factors. Corso et $\mathrm{a}^{37}$ suggested that BPs inhibit the survival of stromal cells and block the interaction of plasma and stromal cells, thus interfering with bone microenvironment. In addition, inhibition of angiogenesis ${ }^{17}$ and immunomodulatory effects of BPs including activation of $\mathrm{V} \gamma 9 \mathrm{~V} \delta 2 \mathrm{~T}$ cell-mediated innate immunity ${ }^{13,35}$ may contribute to their negative effects on tumor progression.

The in-vitro evidence of the antimyeloma effects of BPs was further confirmed by several clinical studies that demonstrate the efficacy of BPs in reducing skeletal events in patients with MM with a concomitant antimyeloma effect. ${ }^{38-42}$ Aviles et a/ ${ }^{41}$ conducted a trial in 2007 and demonstrated that addition of zoledronic acid to conventional chemotherapy in treatmentnaive patients improved 5-year event-free survival and 5-year OS compared with conventional therapy alone. It is of note that in this trial the event-free survival was high with $80 \%$ in the group treated with zoledronic acid. More recently, the randomized, controlled Medical Research Council Myeloma IX study demonstrated that in newly diagnosed patients with MM, combining conventional therapy with zoledronic acid provided a significant survival advantage compared with clodronate, across all treatment pathways. ${ }^{41,42}$ However, the response rates within the intensive and non-intensive chemotherapy arms did not differ with zoledronic acid vs clodronate treatment, suggesting that the zoledronic acidassociated OS advantage occurred independently from the myeloma response. Further, in this trial thalidomide was the only novel agent used in the intensive or non-intensive cohorts. Novel agents such as bortezomib ${ }^{48}$ and lenalidomide ${ }^{49}$ target MM cells and bone marrow microenvironment cells mediating bone formation and resorption. Therefore, it is not surprising that antiresorptive agents that primarily target the bone (that is, BPs such as zoledronic acid and pamidronate) may also favorably impact MM

Future trials need to incorporate novel agents to determine their optimal use as both antimyeloma therapy and their synergy with BPs in terms of controlling bone disease. ${ }^{41,42}$ Ongoing studies such as DAZZLE $(N=53)$ and a larger singlearm trial in Australia (MM6; $N=243$ ) are evaluating the effect of zoledronic acid on disease progression in patients with MM. Data from these studies may provide additional clinical insights into the therapeutic role of zoledronic acid in patients with MM.

Although other studies ${ }^{45-47}$ suggest that BPs do not improve mortality in the overall study population after treatment with BP, the majority of data presented herein provides evidence for the antimyeloma effects of BPs. Further, several studies have proven the reduction of skeletal-related events in $\mathrm{MM}$ patients treated with either pamidronate or zoledronic acid. ${ }^{50}$ Nevertheless, based on the fact that BPs increase the risk of osteonecrosis of jaw and are possibly associated with increased risk of atypical subtrochanteric fractures, ${ }^{51}$ the use of BPs needs to be critically evaluated in the context of the clinical situation of each individual patient. Although the optimal duration of BP treatment in this patient population is unknown, the potential antimyeloma benefit warrants further evaluation of the risk: benefit ratio of BPs in conjunction with primary treatment for MM. Thus, further clinical studies that assess the efficacy of BPs in combination with the prevailing standards of care are warranted.

\section{Conflict of interest}

The authors declare no conflict of interest.

\section{Acknowledgements}

Financial support for medical editorial assistance was provided by Novartis Pharmaceuticals. We thank Jerome Sah, PhD, ProEd Communications, Inc., for his medical editorial assistance with this manuscript.

\section{References}

1 Raab MS, Podar K, Breitkreutz I, Richardson PG, Anderson KC. Multiple myeloma. Lancet 2009; 374: 324-339.

2 Corso A, Ferretti E, Lazzarino M. Zoledronic acid exerts its antitumor effect in multiple myeloma interfering with the bone marrow microenvironment. Hematology 2005; 10: 215-224.

3 Chauhan D, Uchiyama H, Akbarali Y, Urashima M, Yamamoto K, Libermann TA et al. Multiple myeloma cell adhesion-induced interleukin- 6 expression in bone marrow stromal cells involves activation of NF-kappa B. Blood 1996; 87: 1104-1112.

4 Hideshima T, Bergsagel PL, Kuehl WM, Anderson KC. Advances in biology of multiple myeloma: clinical applications. Blood 2004; 104: 607-618.

5 Kuehl WM, Bergsagel PL. Multiple myeloma: evolving genetic events and host interactions. Nat Rev Cancer 2002; 2: 175-187.

6 Fleisch H. Development of bisphosphonates. Breast Cancer Res 2002; 4: 30-34.

7 Russell RG, Xia Z, Dunford JE, Oppermann U, Kwaasi A, Hulley PA et al. Bisphosphonates: an update on mechanisms of action and how these relate to clinical efficacy. Ann NY Acad Sci 2007; 1117: 209-257.

8 Luckman SP, Coxon FP, Ebetino FH, Russell RG, Rogers MJ. Heterocycle-containing bisphosphonates cause apoptosis and inhibit bone resorption by preventing protein prenylation: evidence from structure-activity relationships in $\mathrm{J774}$ macrophages. J Bone Miner Res 1998; 13: 1668-1678.

9 Luckman SP, Hughes DE, Coxon FP, Graham R, Russell G, Rogers MJ. Nitrogen-containing bisphosphonates inhibit the mevalonate pathway and prevent post-translational prenylation of GTP-binding proteins, including Ras. J Bone Miner Res 1998; 13: 581-589.

10 Drake MT, Clarke BL, Khosla S. Bisphosphonates: mechanism of action and role in clinical practice. Mayo Clin Proc 2008; 83: 1032-1045.

11 Clezardin P. Bisphosphonates' antitumor activity: an unravelled side of a multifaceted drug class. Bone 2011; 48: 71-79.

12 Guenther A, Gordon S, Tiemann M, Burger R, Bakker F, Green JR et al. The bisphosphonate zoledronic acid has antimyeloma activity in vivo by inhibition of protein prenylation. Int J Cancer 2010; 126: 239-246.

13 Kunzmann V, Bauer E, Feurle J, Weissinger F, Tony HP, Wilhelm M. Stimulation of gammadelta $T$ cells by aminobisphosphonates and induction of antiplasma cell activity in multiple myeloma. Blood 2000; 96: 384-392.

14 Moschetta M, Di Pietro G, Ria R, Gnoni A, Mangialardi G, Guarini A et al. Bortezomib and zoledronic acid on angiogenic 
and vasculogenic activities of bone marrow macrophages in patients with multiple myeloma. Eur J Cancer 2010; 46: 420-429.

15 Ribatti D, Nico B, Vacca A. Importance of the bone marrow microenvironment in inducing the angiogenic response in multiple myeloma. Oncogene 2006; 25: 4257-4266.

16 Ribatti D, Vacca A. The role of monocytes-macrophages in vasculogenesis in multiple myeloma. Leukemia 2009; 23: 1535-1536.

17 Santini D, Vincenzi B, Galluzzo S, Battistoni F, Rocci L, Venditti O et al. Repeated intermittent low-dose therapy with zoledronic acid induces an early, sustained, and long-lasting decrease of peripheral vascular endothelial growth factor levels in cancer patients. Clin Cancer Res 2007; 13 (15 Pt 1): 4482-4486.

18 Scavelli C, Nico B, Cirulli T, Ria R, Di Pietro G, Mangieri D et al. Vasculogenic mimicry by bone marrow macrophages in patients with multiple myeloma. Oncogene 2008; 27: 663-674.

19 Baulch-Brown C, Molloy TJ, Yeh SL, Ma D, Spencer A. Inhibitors of the mevalonate pathway as potential therapeutic agents in multiple myeloma. Leuk Res 2007; 31: 341-352.

20 Shipman CM, Croucher PI, Russell RG, Helfrich MH, Rogers MJ. The bisphosphonate incadronate (YM175) causes apoptosis of human myeloma cells in vitro by inhibiting the mevalonate pathway. Cancer Res 1998; 58: 5294-5297.

21 Shipman CM, Rogers MJ, Apperley JF, Russell RG, Croucher PI. Bisphosphonates induce apoptosis in human myeloma cell lines: a novel anti-tumour activity. Br J Haematol 1997; 98: 665-672.

22 Drexler HG, MacLeod RA, Dirks WG. Cross-contamination: HS-Sultan is not a myeloma but a Burkitt lymphoma cell line. Blood 2001; 98: 3495-3496.

23 Roelofs AJ, Hulley PA, Meijer A, Ebetino FH, Russell RG, Shipman $\mathrm{CM}$. Selective inhibition of Rab prenylation by a phosphonocarboxylate analogue of risedronate induces apoptosis, but not S-phase arrest, in human myeloma cells. Int J Cancer 2006; 119: $1254-1261$

24 Lawson MA, Coulton L, Ebetino FH, Vanderkerken K, Croucher PI. Geranylgeranyl transferase type II inhibition prevents myeloma bone disease. Biochem Biophys Res Commun 2008; 377: 453-457.

25 Croucher PI, De Hendrik R, Perry MJ, Hijzen A, Shipman CM, Lippitt J et al. Zoledronic acid treatment of 5T2MM-bearing mice inhibits the development of myeloma bone disease: evidence for decreased osteolysis, tumor burden and angiogenesis, and increased survival. J Bone Miner Res 2003; 18: 482-492.

26 Monkkonen H, Auriola S, Lehenkari P, Kellinsalmi M, Hassinen IE, Vepsalainen J et al. A new endogenous ATP analog (Apppl) inhibits the mitochondrial adenine nucleotide translocase (ANT) and is responsible for the apoptosis induced by nitrogen-containing bisphosphonates. Br J Pharmacol 2006; 147: 437-445.

27 Raikkonen J, Monkkonen H, Auriola S, Monkkonen J. Mevalonate pathway intermediates downregulate zoledronic acid-induced isopentenyl pyrophosphate and ATP analog formation in human breast cancer cells. Biochem Pharmacol 2010; 79: 777-783.

28 Schmidmaier R, Simsek M, Baumann P, Emmerich B, Meinhardt G. Synergistic antimyeloma effects of zoledronate and simvastatin. Anticancer Drugs 2006; 17: 621-629.

29 Tassone P, Forciniti S, Galea E, Morrone G, Turco MC, Martinelli V et al. Growth inhibition and synergistic induction of apoptosis by zoledronate and dexamethasone in human myeloma cell lines. Leukemia 2000; 14: 841-844.

30 Ural AU, Yilmaz MI, Avcu F, Pekel A, Zerman M, Nevruz O et al. The bisphosphonate zoledronic acid induces cytotoxicity in human myeloma cell lines with enhancing effects of dexamethasone and thalidomide. Int J Hematol 2003; 78: 443-449.

31 Casetti R, Martino A. The plasticity of gamma delta $T$ cells: innate immunity, antigen presentation and new immunotherapy. Cell Mol Immunol 2008; 5: 161-170.

32 Fiore F, Castella B, Nuschak B, Bertieri R, Mariani S, Bruno B et al. Enhanced ability of dendritic cells to stimulate innate and adaptive immunity upon short-term incubation with zoledronic acid. Blood 2007; 110: 921-927.

33 Sato K, Kimura S, Segawa H, Yokota A, Matsumoto S, Kuroda J et al. Cytotoxic effects of gammadelta T cells expanded ex vivo by a third generation bisphosphonate for cancer immunotherapy. Int J Cancer 2005; 116: 94-99.

34 Yuasa T, Sato K, Ashihara E, Takeuchi M, Maita S, Tsuchiya N et al. Intravesical administration of gammadelta $T$ cells success- fully prevents the growth of bladder cancer in the murine model. Cancer Immunol Immunother 2009; 58: 493-502.

35 Abe Y, Muto M, Nieda M, Nakagawa Y, Nicol A, Kaneko T et al. Clinical and immunological evaluation of zoledronate-activated Vgamma9gammadelta T-cell-based immunotherapy for patients with multiple myeloma. Exp Hematol 2009; 37: 956-968.

36 Coscia M, Quaglino E, lezzi M, Curcio C, Pantaleoni F, Riganti C et al. Zoledronic acid repolarizes tumor-associated macrophages and inhibits mammary carcinogenesis by targeting the mevalonate pathway. J Cell Mol Med 2010; 14: 2803-2815.

37 Corso A, Ferretti E, Lunghi M, Zappasodi $\mathrm{P}$, Mangiacavalli S, De Amici $\mathrm{M}$ et al. Zoledronic acid down-regulates adhesion molecules of bone marrow stromal cells in multiple myeloma: a possible mechanism for its antitumor effect. Cancer 2005; 104: 118-125.

38 Berenson JR, Dimopoulos MA, Chen Y-M. Zoledronic acid may improve survival compared to pamidronate in patients with $M M$ and high BALP levels: univariate and multivariate models of hazard ratios. Blood 2006; 108 Abstract 3589.

39 Berenson JR, Lichtenstein A, Porter L, Dimopoulos MA, Bordoni R, George $\mathrm{S}$ et al. Long-term pamidronate treatment of advanced multiple myeloma patients reduces skeletal events. Myeloma Aredia Study Group. J Clin Oncol 1998; 16: 593-602.

40 McCloskey EV, Dunn JA, Kanis JA, MacLennan IC, Drayson MT. Long-term follow-up of a prospective, double-blind, placebocontrolled randomized trial of clodronate in multiple myeloma. Br J Haematol 2001; 113: 1035-1043.

41 Aviles A, Nambo MJ, Neri N, Castaneda C, Cleto S, Huerta-Guzman J. Antitumor effect of zoledronic acid in previously untreated patients with multiple myeloma. Med Oncol 2007; 24: 227-230.

42 Morgan GJ, Davies FE, Gregory WM, Cocks K, Bell SE, Szubert AJ et al. First-line treatment with zoledronic acid as compared with clodronic acid in multiple myeloma (MRC Myeloma IX): a randomised controlled trial. Lancet 2010; 376: 1989-1999.

43 Kellinsalmi M, Monkkonen H, Monkkonen J, Leskela HV, Parikka V, Hamalainen $\mathrm{M}$ et al. In vitro comparison of clodronate, pamidronate and zoledronic acid effects on rat osteoclasts and human stem cellderived osteoblasts. Basic Clin Pharmacol Toxicol 2005; 97: 382-391.

44 Kavanagh KL, Guo K, Dunford JE, Wu X, Knapp S, Ebetino FH et al. The molecular mechanism of nitrogen-containing bisphosphonates as antiosteoporosis drugs. Proc Natl Acad Sci USA 2006; 103: 7829-7834.

45 Mhaskar R, Redzepovic J, Wheatley K, Clark OA, Miladinovic B, Glasmacher A et al. Bisphosphonates in multiple myeloma. Cochrane Database Syst Rev (Online) 2010, CD003188.

46 McCloskey EV, MacLennan IC, Drayson MT, Chapman C, Dunn J, Kanis JA. A randomized trial of the effect of clodronate on skeletal morbidity in multiple myeloma. MRC Working Party on Leukaemia in Adults. Br J Haematol 1998; 100: 317-325.

47 Musto P, Petrucci MT, Bringhen S, Guglielmelli T, Caravita T, Bongarzoni $\mathrm{V}$ et al. A multicenter, randomized clinical trial comparing zoledronic acid versus observation in patients with asymptomatic myeloma. Cancer 2008; 113: 1588-1595.

48 Giuliani N, Morandi F, Tagliaferri S, Lazzaretti M, Bonomini S, Crugnola $\mathrm{M}$ et al. The proteasome inhibitor bortezomib affects osteoblast differentiation in vitro and in vivo in multiple myeloma patients. Blood 2007; 110: 334-338.

49 Kumar S, Giralt S, Stadtmauer EA, Harousseau JL, Palumbo A, Bensinger $\mathrm{W}$ et al. Mobilization in myeloma revisited: IMWC consensus perspectives on stem cell collection following initial therapy with thalidomide-, lenalidomide-, or bortezomib-containing regimens. Blood 2009; 114: 1729-1735.

50 Berenson JR, Lichtenstein A, Porter L, Dimopoulos MA, Bordoni R, George $\mathrm{S}$ et al. Efficacy of pamidronate in reducing skeletal events in patients with advanced multiple myeloma. Myeloma Aredia Study Group. N Engl J Med 1996; 334: 488-493.

51 Puhaindran ME, Farooki A, Steensma MR, Hameed M, Healey JH, Boland PJ. Atypical subtrochanteric femoral fractures in patients with skeletal malignant involvement treated with intravenous bisphosphonates. J Bone Joint Surg Am 2011; 93: 1235-1242.

This work is licensed under the Creative Commons Attribution-NonCommercial-No Derivative Works 3.0 Unported License. To view a copy of this license, visit http:// creativecommons.org/licenses/by-nc-nd/3.0/ 